Article

\title{
Developing Computational Model to Predict Protein-Protein Interaction Sites Based on the XGBoost Algorithm
}

\author{
Aijun Deng ${ }^{1,2,3,+}$, Huan Zhang ${ }^{4}+$, Wenyan Wang ${ }^{4}$, Jun Zhang ${ }^{5}$, Dingdong Fan ${ }^{2}$, \\ Peng Chen ${ }^{5, *(D)}$ and Bing Wang $1,4,5, *$ \\ 1 Key Laboratory of Metallurgical Emission Reduction \& Resources Recycling (Anhui University of \\ Technology), Ministry of Education, Ma'anshan 243002, China; ajdeng@163.com \\ 2 School of Metallurgical Engineering, Anhui University of Technology, Ma'anshan 243032, China \\ 3 Department of Engineering, University of Leicester, Leicester LE1 7RH, UK \\ 4 School of Electrical and Information Engineering, Anhui University of Technology, Ma'anshan 243032, \\ China; 1135130589@163.com (H.Z.); 1743388711@163.com (W.W.) \\ 5 Co-Innovation Center for Information Supply \& Assurance Technology, Anhui University, Hefei 230032, \\ China; wwwzhangjun@ahu.edu.cn \\ * Correspondence: pchen@ahu.edu.cn (P.C.); wangbing@ustc.edu (B.W.) \\ + These authors contributed equally to this work.
}

Received: 3 February 2020; Accepted: 23 March 2020; Published: 25 March 2020

check for updates

\begin{abstract}
The study of protein-protein interaction is of great biological significance, and the prediction of protein-protein interaction sites can promote the understanding of cell biological activity and will be helpful for drug development. However, uneven distribution between interaction and non-interaction sites is common because only a small number of protein interactions have been confirmed by experimental techniques, which greatly affects the predictive capability of computational methods. In this work, two imbalanced data processing strategies based on XGBoost algorithm were proposed to re-balance the original dataset from inherent relationship between positive and negative samples for the prediction of protein-protein interaction sites. Herein, a feature extraction method was applied to represent the protein interaction sites based on evolutionary conservatism of proteins, and the influence of overlapping regions of positive and negative samples was considered in prediction performance. Our method showed good prediction performance, such as prediction accuracy of 0.807 and MCC of 0.614 , on an original dataset with 10,455 surface residues but only 2297 interface residues. Experimental results demonstrated the effectiveness of our XGBoost-based method.
\end{abstract}

Keywords: protein interaction sites; unbalanced data sets; overlapping regions; XGBoost

\section{Introduction}

Protein-protein interaction (PPI) is the main way to realize the regulation of biological information, and it is an important factor to determine the fate of cells [1-3]. The study of protein-protein interactions is the basis for understanding life activities and one of the most important topics in the post-genome era. With the completion of the human genome project, the data in the protein sequence database has increased dramatically, and the number of protein structures and PPIs are much in arrear of that of sequences. Identification of protein-protein interaction sites (PPIS) by experimental methods is not only time-consuming and laborious, but also suffers from high false positives and negatives.

Fortunately, predicting protein-protein interaction sites using computational methods has become a hot topic with the development of machine learning algorithms [4-8]. Previous studies showed that support vector machine (SVM) and its improved methods can predict effectively protein interaction 
sites [9-14]. Computational algorithms such as random forests, KNN, and Naive Bayes Classifier have been also applied to the prediction of PPIs [15-18]. Wang et al. proposed a new method for predicting protein interaction sites in hetero-complexes using a radial basis function neural network (RBFNN) set model, which uses only evolutionary conservation information and spatial sequence profile of proteins, and achieved a good predictive result [19].

However, only a small number of protein interaction sites were experimentally validated within current databases, which causes a highly imbalanced distribution of interaction or non-interaction sites, and therefore decreases the predictive performance of computational models in predicting protein functional sites. There are some works that tried to address the problem of sample imbalance. Li proposed RBFIS (improved radial basis function by SMOTE) to solve the problem of sample imbalance [20]. The down-sampling methods based on easy ensemble and balance cascade were proposed to solve the problem of unbalanced data [21]. Wang et al. proposed a manifold learning approach, and tried to re-balance the original dataset using clustering algorithm [22]. Although these methods have made great progress in predicting protein interaction sites, the random down-sampling they adopted may result in the missing of some important sample information, and the risk of overfitting in the prediction of protein interaction sites.

In this work, two imbalanced data processing strategies based on XGBoost algorithm were proposed to re-balance the original dataset from inherent relationship between positive and negative samples in current dataset, which can effectively reduce the influence of data imbalance problem, and therefore improve the prediction performance of protein interaction sites. Our work focuses on the extraction of related feature attributes for negative samples selection, and applies XGBoost algorithm to improve the prediction performance of protein interaction sites, which is a very effective machine learning algorithm can handle large-scale data efficiently [23]. The main contributions of this study are to extract the characteristics of amino acids, which can reduce the data dimension and increase the speed of operation, and propose two down-sampling methods, i.e., repetitive nearest neighbor rule (RENN) and instance hardness threshold (IHT), to select non-interface residues with high reliability. Experimental results showed that our proposed method achieved a good prediction performance, such as prediction accuracy of $80.7 \%$ and sensitivity of $81.2 \%$.

\section{Results}

The goal of this study is to identify interaction sites on the surface of proteins and build predictors using a computational method. The data set used herein is the 91 non-redundant protein chains which have a total of 10,455 surface residues; 2297 of them are interfacial residues and 8158 non-interface residues. The work builds a balanced data set from the original unbalance one to improve the prediction performance of protein interaction sites from protein sequences.

\subsection{Evaluation Criteria}

Traditional machine learning algorithms usually use accuracy as an evaluation index. However, for an imbalanced dataset, the evaluation of classifiers tended to reduce the classification effect of a few types of samples. Therefore, multiple evaluation indicators, such as accuracy (Acc), sensitivity (Sen), precision (Pre) and specificity (Spe), are used to evaluate the prediction results in this study. As an effective indicator for the classification performance of unbalanced data sets, $F-$ measure is a weighted harmonic mean of precision and sensitivity. Matthews correlation coefficient (MCC) describes the degree of correlation between the predicted and actual residue classification, and its value ranges from -1 to 1 , where -1 indicates the worst prediction, and 1 the best prediction.

$$
\begin{gathered}
A c c=\frac{T P+T N}{T P+F P+T N+F N} \\
\operatorname{Sen}=\frac{T P}{T P+F N}
\end{gathered}
$$




$$
\begin{gathered}
\text { Pre }=\frac{T P}{T P+F P} \\
\text { Spe }=\frac{T N}{F P+T N} \\
F-\text { measure }=2 \times \frac{\text { Pre } \times \text { Sen }}{\text { Pre }+ \text { Sen }} \\
M C C=\frac{(T P \times T N-F P \times F N)}{\sqrt{(T P+F P) \times(T P+F N) \times(T N+F P) \times(T N+F N)}}
\end{gathered}
$$

where TP represents the number of interfacial residues that are actually interfacial residues, FP represents the number of interfacial residues that are actually non-interface residues. TN represents the number of non-interface residues that are predicted to be non-interface residues, and FN indicates the number of non-interface residues that are actually interfacial residues.

\subsection{Predictive Performance in Two Balanced Modes}

Although the purpose of RENN and IHT is same, the balance strategy is different. RENN will repeatedly remove the noise of non-interface residues and overlapping areas of samples until it cannot be removed. IHT can only remove non-interface residues and achieve a balance with interface residues. The number of samples of data processed by two sampling methods is shown in Table 1.

Table 1. Sample numbers within the original and re-balance datasets.

\begin{tabular}{ccc}
\hline & \multicolumn{2}{c}{ Samples } \\
\cline { 2 - 3 } & Positive & Negative \\
\hline Original data & 2297 & 8158 \\
RENN & 2297 & 2131 \\
IHT & 2297 & 2297 \\
\hline
\end{tabular}

Table 2 shows the performance of IHT and RENN in predicting protein-protein interaction sites. It can be seen that both methods can effectively predict protein-protein interaction sites, i.e., 0.707 of accuracy for RENN and 0.807 for IHT when they are combined with XGBoost predictor. It can be found that a relatively high MCC values for both predictors show the effectiveness of the features extracted from evolutionary conservation in differential interface residues from non-interface ones. With Comparison to RENN, IHT shows a better prediction, which can achieve $9.94 \%$ in accuracy, $4.66 \%$ in sensitivity and $7.69 \%$ in F-score in the performance measures.

Table 2. The prediction performance of two re-balance models.

\begin{tabular}{ccccccc}
\hline & Acc & F-measure & Pre & Spe & Sen & MCC \\
\hline RENN-XGB & 0.707 & 0.731 & 0.699 & 0.645 & 0.765 & 0.454 \\
IHT-XGB & 0.807 & 0.808 & 0.804 & 0.802 & 0.812 & 0.614 \\
\hline
\end{tabular}

From the experimental results, it can be seen that our hypothesis there are a large number of sample overlap areas within the data set is correct. Although both of RENN and IHT can re-balance the original dataset, IHT is more efficient in processing sample overlap areas, which may be the main reason a better prediction performance can be achieved based on a similar number of positives and negatives. According to specificity and sensitivity, IHT-XGB can improve the recognition rate of a few samples when that of most samples does not decrease.

\subsection{Comparison of Unbalanced and Balanced Data Sets}

To infer the effect of dataset balance, we compare the results by the same XGBoost predictor on the original unbalanced dataset and balanced dataset using RENN and IHT methods. Experimental 
results show that when the data sets are unbalanced, the accuracy of the model reaches 0.78 , but the sensitivity is only 0.0021 , and the F value is 0.0042 . The very low value of sensitivity shows that only a very small number of positives can be identified, and most of predictions are negative, which means that prediction results tend to the powerful categories within an imbalanced dataset, especially in the original dataset in this work where the negative samples far exceed the positive samples. The highest specificity on the original imbalanced dataset, such as almost equal to 1 , shows almost all of negatives can be predicted, which further demonstrated the overfitting problem for unbalanced data processing. The comparison of prediction performance on the original unbalanced and re-balanced dataset can be found in Figure 1.

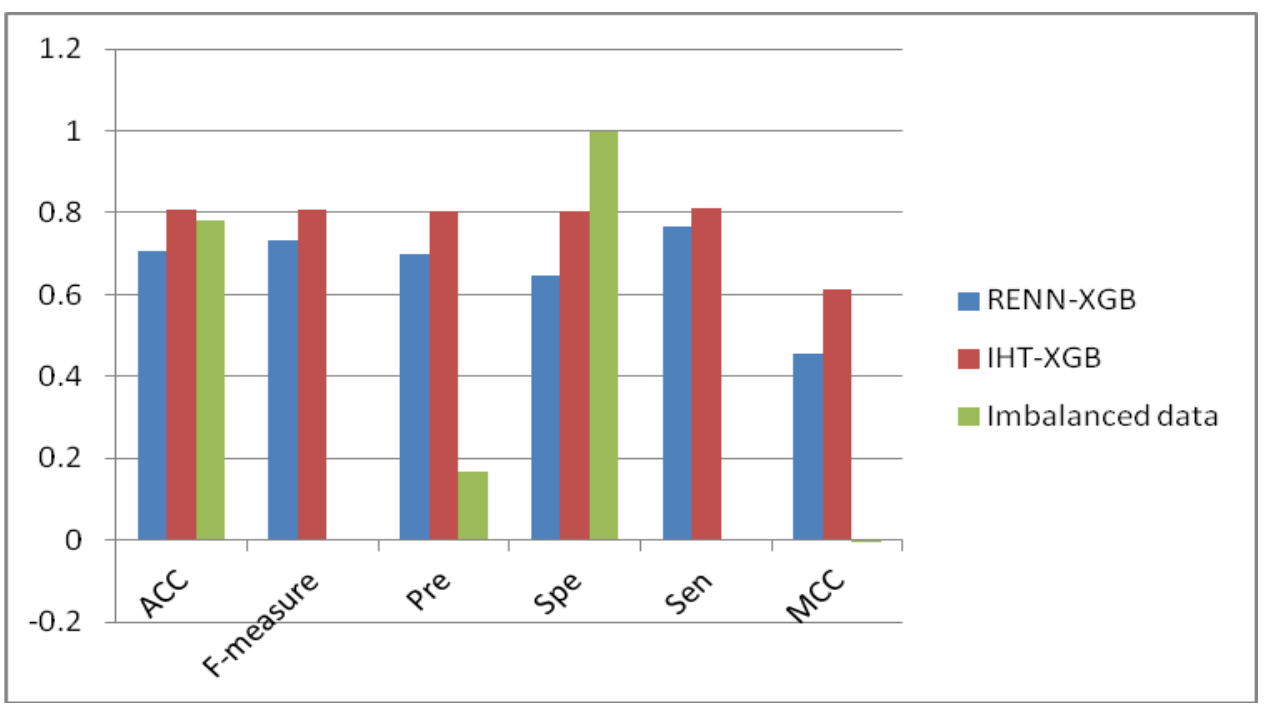

Figure 1. Performance comparison between the original and re-balanced datasets.

Detailed information about the predictions on the balanced dataset based on the two down-sampling methods proposed in this work and the original dataset can be seen in Table 3 . It can be found that the number of negative samples is much larger than that of positive samples, and the number of TN and FN is much larger than that of TP and FP. After classification, the number of negative samples is 346 times that of positive samples. For the balanced data set after sample sampling, the proportion of negative samples and positive samples after classification is equivalent, and the prediction effect is far better than that of unbalanced data set.

Table 3. Classification results of two sampling methods and imbalance dataset.

\begin{tabular}{ccccccc}
\hline & \multicolumn{3}{c}{ Sample } & \multicolumn{4}{c}{ Results } \\
\cline { 2 - 7 } & Positive & Negative & TP & TN & FP & FN \\
\hline Imbalanced data & 2297 & 8158 & 5 & 8151 & 25 & 2249 \\
RENN & 2131 & 2297 & 1758 & 1376 & 755 & 539 \\
IHT & 2291 & 2297 & 1864 & 1844 & 454 & 432 \\
\hline
\end{tabular}

\section{Discussion}

\subsection{Comparison with Other Methods}

To analyze the performance of our proposed methods, we compared the prediction results with other approaches in protein interaction sites identification. To make the comparison more convincing, this work compared the prediction performance of the presented re-balanced strategies with four previous studies using the same dataset. Wang's work extracted the residue evolutionary conservation and sequence profile to infer protein interaction sites, which is already a benchmark 
method for the prediction performance comparison [24]. Kuo and Li developed an SVM model to predict protein-protein interaction sites by extracting five different sequence features [25]. Liu et al. identified protein-protein interaction sites with temperature factor, sequence profile and accessible surface area [26]. Mei et al. tried to predict protein-protein interaction sites by using semi-supervised SVMs [27]. All of these four methods randomly selected subset of non-interacting sites in the prediction. The performance comparison of five works is shown in Figure 2.

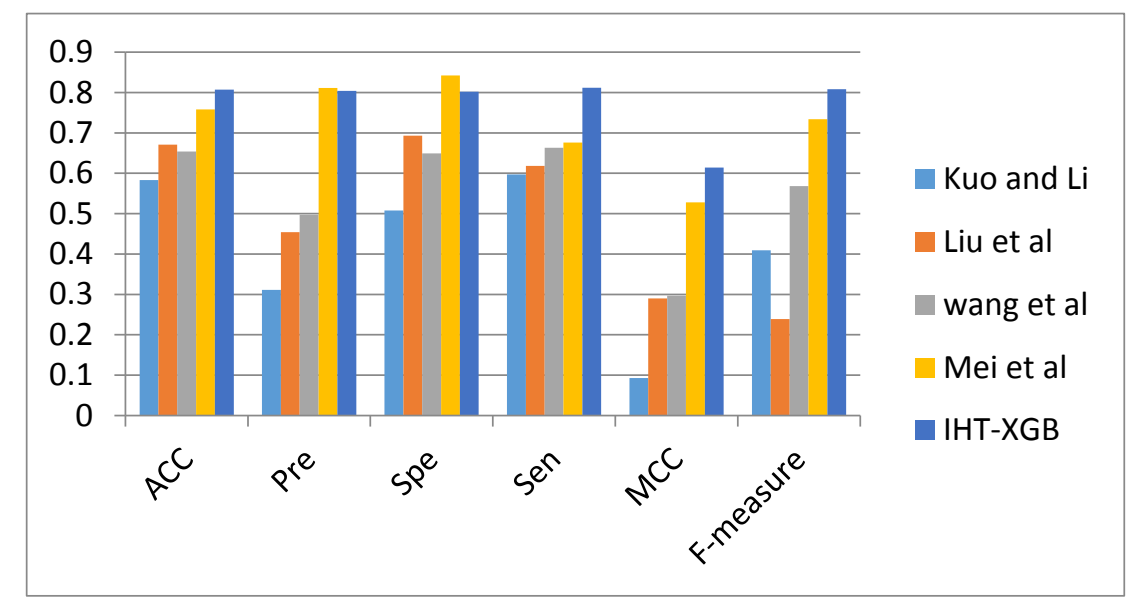

Figure 2. Prediction performance comparison of IHT-XGB method with four previous works.

It can be seen from Figure 2 that the proposed IHT-XGB method can achieve a better overall performance than the other four approaches can do. For all of the methods, IHT-XGB is not only as good as Mei's work on the measures of Pre and Spe, and can outperform all of the other four measures. The accuracy of IHT-XGB is $80.71 \%$, which is $4.9 \%$ higher than that of Mei's, $15.3 \%$ than Wang's, $13.6 \%$ than Liu's, and 22.4\% than Li and Kuo's work. The MCC of IHT-XGB is 0.614 indicates that the features used in predictors can distinguish interaction and non-interaction residues effectively. The high Sen of 0.812 shows that IHT-XGB can identify more protein interface residues from protein chains. Furthermore, it can be found that the value of IHT-XGB on the five measures, i.e., ACC, Sen, Spe, Pre, and F-measure are very close, which indicated that the accurate prediction rate in positives and negatives are also close, and there is no tendency for the predictor to overfitting to the majority class.

In this work, the features of residues used herein are similar to that in Kou and Li's work, but the performance are totally different, and the comparison results shows that data re-balance is necessary and effective. In addition, the experimental results also demonstrated our hypothesis that there are a large number of overlapping regions between the positives and negatives in the original dataset. If the overlapping regions of samples were reasonably processed, such as in the case of IHT-XGB, the prediction performance of the model can be improved dramatically.

\subsection{Prediction Performance in Independent Benchmark Datasets}

To further evaluate the prediction performance of IHT-XGB model, three widely used datasets are used for independent testing. All of them are publicly available from previous studies: Dset_186, Dset_72 and Dset_164 [28-30]. These datasets consist of 186, 72, and 164 protein sequences, where the number of interaction sites is 1923, 5517, and 6069 within them, respectively. All of the protein sequences are extracted from PDB database with following the same rules, such as less than $25 \%$ sequence homology identity, less than $3.0 \AA$ of the resolution in X-ray crystallography, and removing transmembrane proteins. The prediction performance of the proposed model is shown in Table 4. It can be found that the values of six measures achieved in these three independent benchmark datasets are lower than that in the dataset used in this work, but it is reasonable because the IHT-XGB model is built on this original dataset. 
Table 4. Prediction performance in benchmark datasets.

\begin{tabular}{cccccccc}
\hline \multirow{5}{*}{ Dset_186 } & Method & Acc & F-measure & Pre & Spe & Sen & MCC \\
\hline & SSWRF & 0.679 & 0.386 & 0.322 & 0.697 & 0.581 & 0.234 \\
& LORIS & 0.604 & 0.384 & 0.287 & 0.586 & $\mathbf{0 . 6 9 8}$ & 0.221 \\
& PSIVER & 0.673 & 0.353 & 0.306 & 0.743 & 0.416 & 0.151 \\
& SCRIBER & 0.78 & 0.279 & 0.279 & 0.87 & 0.279 & 0.15 \\
& DELPHI & $\mathbf{0 . 8 0 3}$ & 0.353 & 0.353 & $\mathbf{0 . 8 8 4}$ & 0.352 & 0.235 \\
& IHT_XGB & 0.716 & $\mathbf{0 . 6 9 4}$ & $\mathbf{0 . 7 5 3}$ & 0.788 & 0.644 & $\mathbf{0 . 4 3 7}$ \\
\hline \multirow{5}{*}{ Dset_72 } & SSWRF & 0.648 & 0.351 & 0.267 & 0.643 & 0.654 & 0.224 \\
& LORIS & 0.614 & 0.324 & 0.238 & 0.610 & 0.631 & 0.177 \\
& PSIVER & 0.661 & 0.278 & 0.25 & 0.693 & 0.465 & 0.135 \\
& SCRIBER & 0.837 & 0.232 & 0.232 & 0.909 & 0.232 & 0.141 \\
& DELPHI & $\mathbf{0 . 8 4 7}$ & 0.275 & 0.276 & $\mathbf{0 . 9 1 5}$ & 0.274 & 0.189 \\
& IHT_XGB & 0.702 & $\mathbf{0 . 6 8 9}$ & $\mathbf{0 . 7 2 1}$ & 0.741 & $\mathbf{0 . 6 6 3}$ & $\mathbf{0 . 4 0 5}$ \\
\hline \multirow{5}{*}{ Dset_164 } & SSWRF & 0.621 & 0.365 & 0.323 & 0.656 & 0.527 & 0.152 \\
& LORIS & 0.588 & 0.323 & 0.263 & 0.609 & 0.538 & 0.111 \\
& PSIVER & 0.596 & 0.295 & 0.253 & 0.634 & 0.464 & 0.078 \\
& SCRIBER & 0.756 & 0.327 & 0.327 & 0.851 & 0.327 & 0.179 \\
& DELPHI & $\mathbf{0 . 7 5 8}$ & 0.332 & 0.332 & $\mathbf{0 . 8 5 2}$ & 0.332 & 0.184 \\
& IHT_XGB & 0.733 & $\mathbf{0 . 7 1 5}$ & $\mathbf{0 . 7 6 7}$ & 0.795 & $\mathbf{0 . 6 7 1}$ & $\mathbf{0 . 4 7 0}$ \\
\hline
\end{tabular}

Table 4 also shows the prediction performance of five models, i.e., SSWRF, LORIS, PSIVER, SCRIBER, and DELPHI, in identification of protein-protein interaction sites [31-37]. The highest results in each performance measures for different models are highlighted as bold type. It can be seen that the IHT_XGB model can achieve the highest values in F-measure, Pre and MCC in all of three datasets, and the best Sen in Dset_72 and Dset_164. The proposed model also achieves the second highest performance of Spe in three datasets and the second Sen in Dset_186. Although DELPHI can get the best Acc and Spe, the other measures are obviously lower than what IHT_XGB can reach. The Sen values are around $0.27-0.35$ in all three benchmark datasets means DELPHI can predict only a small number of protein interaction sites correctly. It can also be found that the IHT_XGB model can get a balanced performance among the different measures, which is similar with it did in the dataset used in this work. The results demonstrated the effectiveness of the proposed model.

\subsection{Visualization of Experimental Results}

To show the results of the proposed methods, a molecular visualization tool, pymol, is adopted to demonstrate our predictions. Figure 3 shows the cartoon and spheres forms of protein chain 1a4y-a and the results of RENN-XGB and IHT-XGB prediction methods. It can be found that by predicting the surface residues involved, our method can improve the overall prediction performance, and successfully predict most interfacial and non-interfacial residues. It can also be seen that IHT-XGB can get better predictions than RENN-XGB.

\subsection{Limits of Prediction Validation}

In this work, the experimental results show that the proposed method can identify interaction sites from protein sequence and outperform other computational algorithms in prediction accuracy. However, those predictions only provide potential options for experimental validation, which can dramatically decrease the number of site candidates, and enhance pertinence of experimental design. Actually, identifying potential interacting sites is only the first step toward understanding the impact of a protein interaction, and the interaction occurs or not is dependent on the binding kinetics of the interaction as well as the environment of the interactions within a cell.

The association rates often play a critical role in the formation of protein-protein, which is determined by the free energy difference of the bonded and non-bonded states, and it is also the 
fundamental theory of protein docking [38]. Theoretically, the strength of a protein-protein interaction can be characterized by a dissociation constant $K_{D}=k_{d} / k_{a}$, where $k_{d}$ is the dissociation rate constant and $k_{a}$ is the association rate constant. Many commonly used techniques provide measurements of $K_{D}$, which can be calculated by the concentration of free proteins, but most of them do not offer the real-time measurements of $k_{d}$ and $k_{a}$ [34].

In future work, the prediction results in this work will be validated by protein-protein docking method. Although the goal of docking is the prediction of three-dimensional structure of the protein complexes using computational modeling methods, the docking areas can provide an important clue for evaluating the accuracy of our prediction. Obviously, if the predicted interaction sites in this work locate onto the interfaces between proteins, they contribute the formation of protein-protein interaction, and therefore have high possibility to be true positive sites.

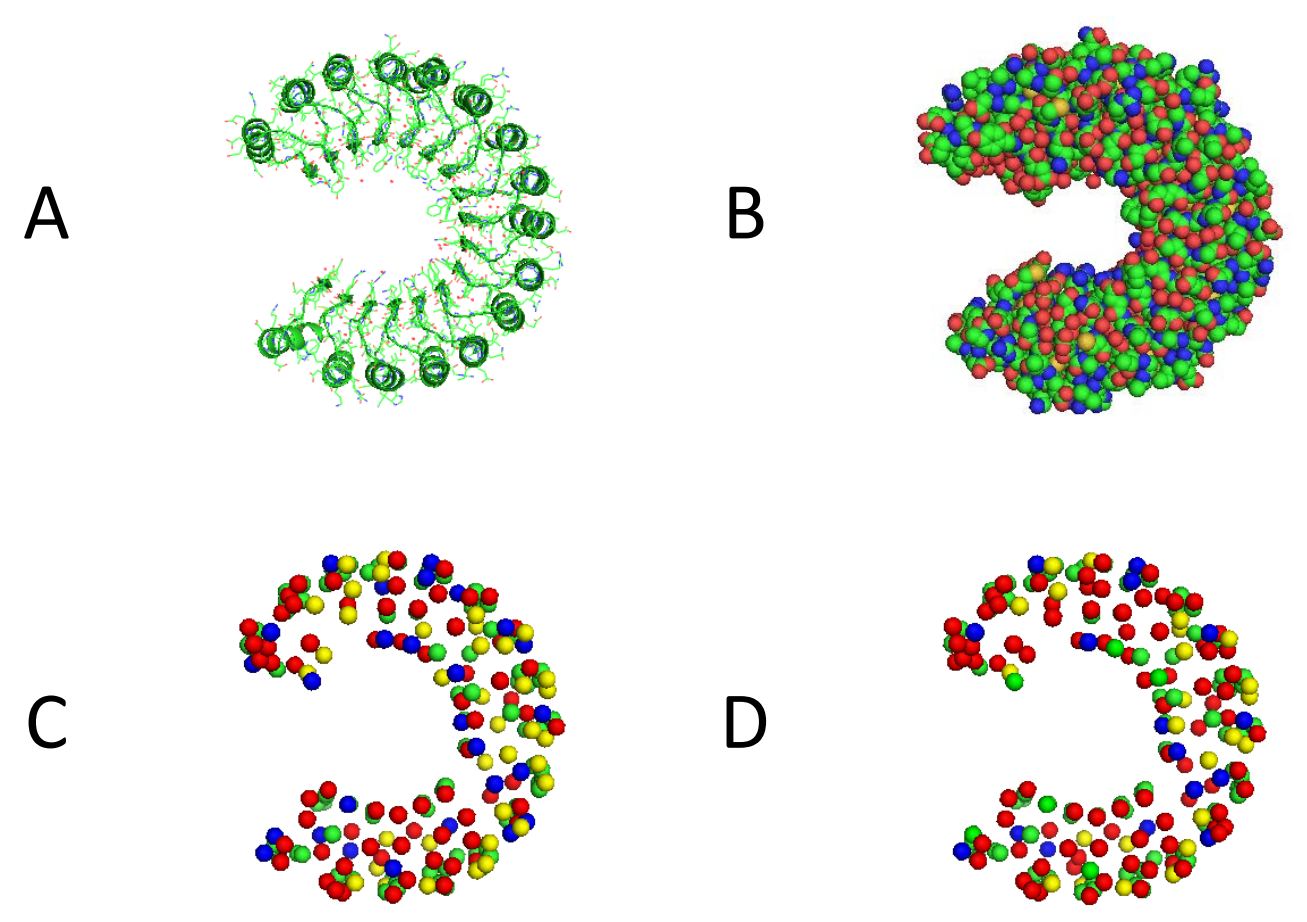

Figure 3. Visualization results of predictions by the proposed methods. (A), (B) represent the cartoon and spheres form of 1a4y-a, and (C), (D) represent predictions based on RENN-XGB and IHT-XGB methods, where green, red, yellow, and blue ball represent the predictions of TP, TN, FP and FN, respectively.

\section{Materials and Methods}

\subsection{Dataset}

Currently, there is no uniform standard dataset for prediction of protein interaction sites due to the lack of corresponding selection criteria when selecting data sets. Therefore, to compare with other research methods, the dataset of this study was screened from the dataset containing 170 transient protein interactions used by Ansari and Helms, which is a gold dataset widely used in protein studies [39]. To ensure the quality of the experimental data, we remove the antibody-antigen interaction, and delete the protein chain pairs with less than 50 residues, leaving only the chain with most interface residues. In addition, the BLASTCLUST program was used to remove proteins with sequence similarity greater than or equal to $30 \%$. Finally, only 91 non-redundant protein chains were left in our work.

The definition of residues is the same as that many previous works adopted $[6,40]$. The relative accessible surface area (RASA) of amino acid residues with maximum accessible surface area of more 
than $16 \%$ is defined as surface residues. Of them, the distance between two residue carbon atoms in the interaction chain greater than $1.2 \mathrm{~nm}$ are defined as non-interface residues, and conversely, defined as interfacial residues. Finally, 91 protein chains in this work produced 10,455 surface residues, of which 2297 were interface residues and 8158 were non-interface residues. Obviously, the data set in an unbalanced data set.

\subsection{Feature Extraction}

In this work, each protein residue is represented by evolutionary conservation scores. A total of five features were extracted based on the evolutionary conservative type of amino acids, such as four features from HSSP database, i.e., residue space sequence, sequence information entropy, relative entropy, and residue sequence weight, as well as one feature, i.e., residue conservative fraction, extracted from Consurf Serve [41].

To take the synergies of neighborhood residues in protein chain, the residue-centered sliding window with 11 lengths and its 10 nearest neighbors on the protein surface are used to encode the residue's eigenvectors. These 10 residues can be processed at the local interface around the target residue, and each of them, just like the target residue, is vectorized into a 24-dimensional feature. Finally, 264-dimensional vectors of each residue are obtained and used to construct future predictors.

\subsection{Unbalanced Data SETS Processing}

In our work, the proportion of positive samples in our dataset is only $21.9 \%$ of the total samples. This imbalance is very common in the studies of protein interaction sites. If a predictive classifier is constructed based on this dataset directly, the model will tend to negative samples, which will lead to inaccurate predictions of positive samples.

From the definitions of the surface and interface on protein chains, there should be some false positives and negatives existing within the original dataset. It can be found that the original dataset was determined by the RASA and the distance of carbon atoms after the protein complexes formed from single chains, which is a hard threshold and cannot totally describe the functional difference between interface and non-interface residues. The number of negative samples is obviously larger than that of positive samples, and there must be a lot of negative samples between positive samples. The hypothesis of our work is that there are a large number of sample overlap areas within the data set, and deduction of the impact of sample overlap can improve the quality of the original dataset, and therefore is of significant for the prediction of protein interaction sites. In this paper, two sample sampling techniques are provided to effectively deal with overlap problems between different categories' protein residues (Figure 4).

\subsubsection{Instance Hardness Threshold}

Instance hardness (IH) was proposed by Michael R. Smith, which can effectively deal with the class overlap problem within data [42]. This method adopted the concept of IH property to represent the probability that the data points are misclassified in the training set. An edge between two or more classes or a data sample with noise characteristics has a higher IH value because the learning algorithm forces them to over-fit. Previous studies showed that IH is derived from Bayes' theorem:

$$
P(\mathrm{~h} \mid \mathrm{t})=\frac{P(\mathrm{t} \mid \mathrm{h}) \mathrm{P}(\mathrm{h})}{P(\mathrm{t})}=\frac{\prod_{i=1}^{|\mathrm{t}|} P\left(\mathrm{x}_{i}, \mathrm{y}_{i} \mid \mathrm{h}\right) \mathrm{P}(\mathrm{h})}{P(\mathrm{t})}=\frac{\prod_{i=1}^{|\mathrm{t}|} P\left(\mathrm{y}_{i} \mid \mathrm{x}_{i}, \mathrm{~h}\right) \mathrm{P}\left(\mathrm{x}_{i} \mid \mathrm{h}\right) \mathrm{P}(\mathrm{h})}{P(\mathrm{t})}
$$

where $\mathrm{h}$ represents a function that maps input features to their associated tags, $\mathrm{t}$ is the training data, $\mathrm{p}\left(y_{i} \mid x_{i}, \mathrm{~h}\right)$ denotes the probability that the mapping function uses the mark $y_{i}$ as the label of the input 
eigenvector $x_{i}$. The larger the $\mathrm{p}\left(y_{i} \mid x_{i}, \mathrm{~h}\right)$ is, the greater the probability that the correct label will be assigned to $x_{i}$. The instance hardness of data point $\left(x_{i}, y_{i}\right)$ can be obtained as:

$$
I H\left(<x_{i}, y_{i}>\right)=1-\mathrm{p}\left(y_{i} \mid x_{i}, \mathrm{~h}\right)
$$

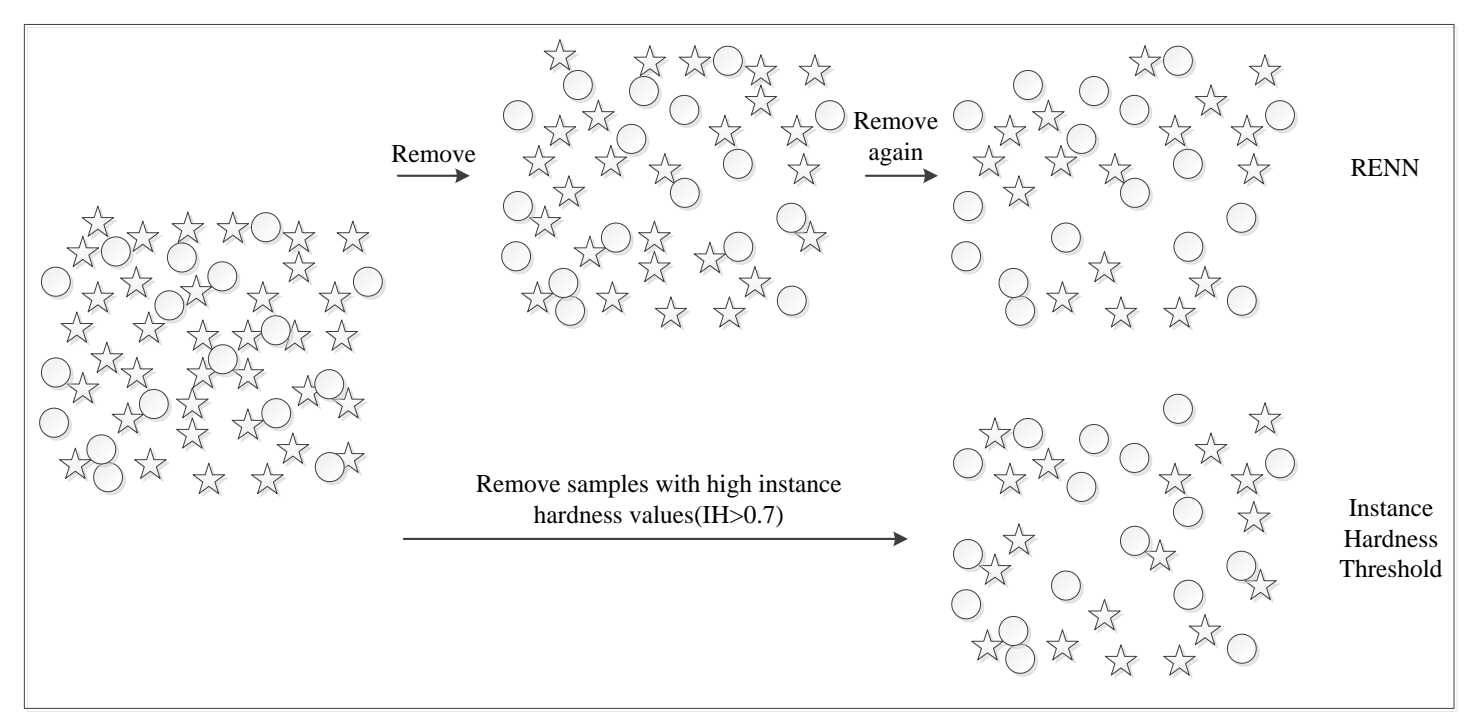

Figure 4. RENN and IHT algorithm schematic. Herein, the circles denote the positive samples, and the stars are negative ones.

The instance hardness threshold (IHT), a downsampling method, can be developed based on this methodology. In IHT, the dataset can be re-balanced by removing the data points with higher IH values in most classes. This data re-balance process requires an estimator to seek a good performance while applying thresholds to remove data points. There are many types of estimators, including random forests [43], decision trees [44], Adaboost, etc. In this work, a logistic regression as estimator, which was proved with higher efficiency and can balance the data set to 1:1 [45]. After the IHT sampling method, the number of negative samples in the data set changed from 8158 to 2297, the same number as the positive samples.

\subsubsection{Repeated Edited Nearest Neighbors}

The repeated edited nearest neighbors (RENN) algorithm is another downsampling method, which can be used to re-balance the original dataset by removing the noise points, and it can be implemented as Algorithm 1. After the RENN [46] sampling, the number of positive samples within the dataset becomes 2131, and the number of negative samples changes from 8158 to 2297.

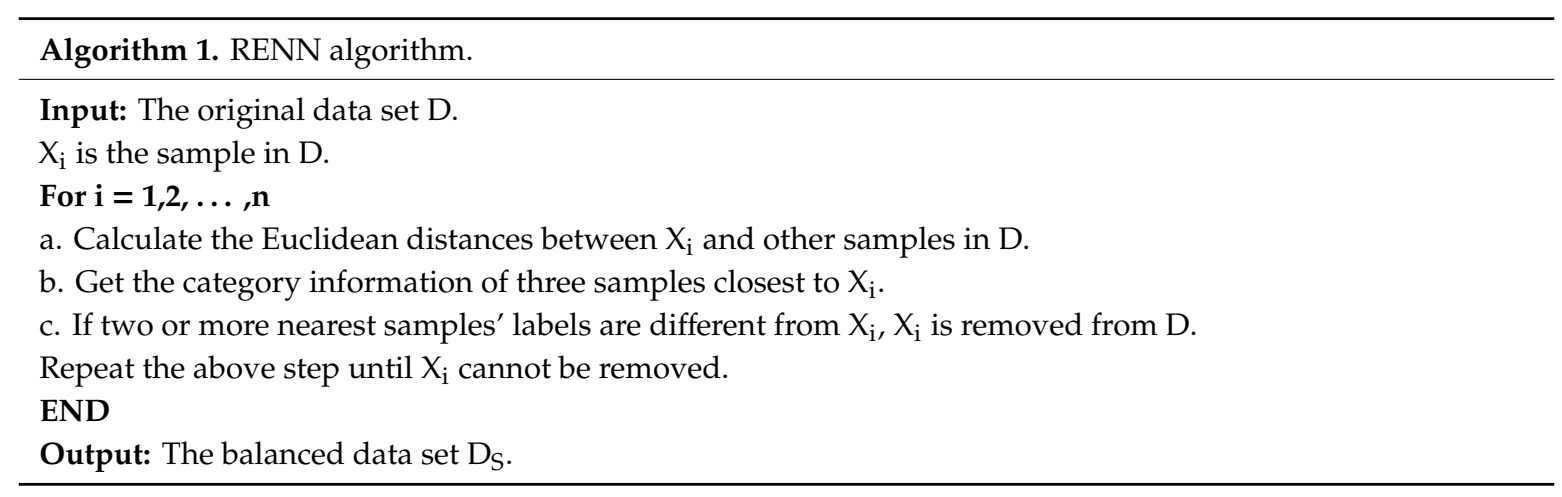




\subsection{XGBoost Algorithm}

The basic idea of XGBoost is that for a given training set $D=\left\{\left(\mathrm{x}_{n}, \mathrm{y}_{n}\right)\right\}_{n=1}^{N}$, the k classifications or regression tree sets trained $F=\left\{\mathrm{f}_{1}(\mathrm{x}), \mathrm{f}_{2}(\mathrm{x}), \ldots, \mathrm{f}_{k}(\mathrm{x})\right\}$ will assign each output sample to different leaf nodes according to the division points of the attribute values, and each leaf node corresponds to one The real-time score $\mathrm{f}_{k}$, when given the sample $\mathrm{x}_{i}$ that needs to be predicted, the prediction result for that sample is the sum of the prediction results of each tree. The specific model is as follows:

$$
\hat{\mathrm{y}}_{i}=\sum_{k=1}^{K} f_{k}\left(\mathrm{x}_{i}\right), f_{k} \in F
$$

where $F$ is the space of all classification trees and regression trees, $\hat{y}_{i}$ corresponds to the prediction result of $x_{i}, f_{k}\left(\mathrm{x}_{i}\right)$ represents the prediction score of leaf nodes obtained after input of sample $\mathrm{x}_{i}$ into kth trees.

The objective function $\mathrm{Obj}(\theta)$ of the model can be defined as:

$$
\operatorname{Obj}(\theta)=\sum_{i=1}^{n} l\left(\mathrm{y}_{i}, \hat{\mathrm{y}}_{i}\right)+\sum_{k=1}^{K} \Omega\left(\mathrm{f}_{k}\right)
$$

where $\theta$ is the parameters of the model. It can be seen that the optimization goal of the model mainly consists of two parts. The first part $\sum_{i=1}^{n} l\left(\mathrm{y}_{i}, \hat{\mathrm{y}}_{i}\right)$ refers to the error function of the model, where the $l(\mathrm{x}, \mathrm{y})$ function is the defined error function. The second part $\sum_{k=1}^{K} \Omega\left(\mathrm{f}_{k}\right)$ is the regularization term of the model, indicating the complexity of the $\mathrm{k}$ tree.

\subsection{Protein Interaction Sites Prediction}

In this work, each surface residue is represented by the feature coding scheme as a 264-dimensional vector, and the original dataset is processed by the two kinds of downsampling algorithms, i.e., RENN and IHT re-balance the number of positives and negatives. Based on the re-balanced dataset, XGBoost is adopted for protein interaction sites prediction. Herein, to ensure the reliability and stability of the prediction results, a 10-fold cross-validation strategy has been used for prediction model construction. In one round of cross-validation, the original dataset is partitioned into 10 subsets, the first one of them is selected as test set, and the other nine as training set, and then the second subset is selected test set, and other nine as a training set, and so on. In one round of cross-validation, each of 10 subsets is used as test set one time, and overall result is adopted to evaluate the predictions. The flowchart of our proposed method can be found in Figure 5. 


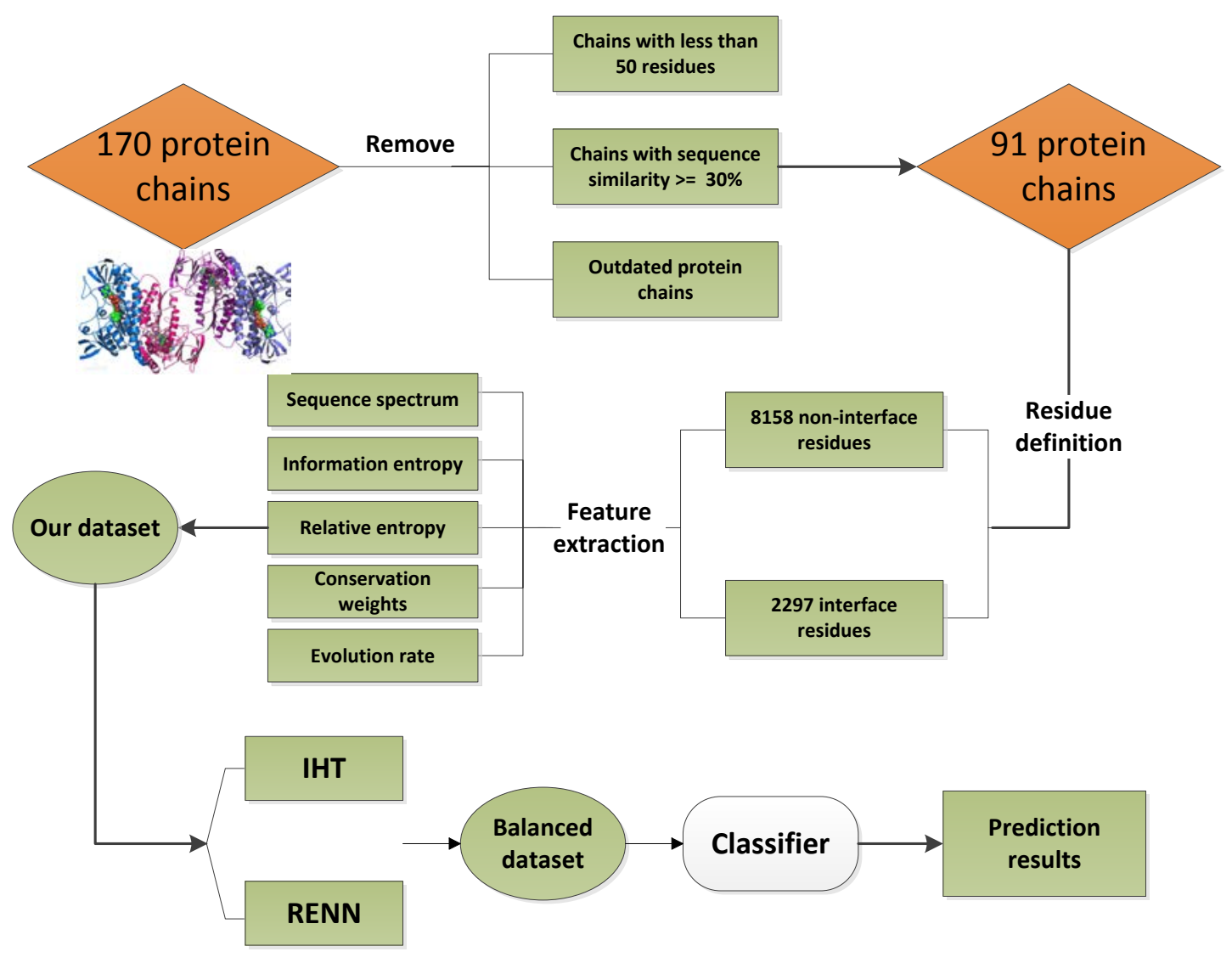

Figure 5. The flowchart of our method.

\section{Conclusions}

This paper presents a method, XGBoost, for predicting protein-protein interaction sites based on unbalanced data processing strategies. Ninety-one data chains were first obtained through a series of processing in the dataset, and 10,455 surface residues were used for the predictor construction. There are 2297 interface residues and 8158 non-interface residues in the original dataset, which is obviously imbalanced. Based on a hypothesis that there is a large number of overlapping data regions in the dataset, two unbalanced data sampling methods, i.e., RENN and IHT, were proposed for processing data overlap regions, and obtain good prediction performance in the classifier. Among them, IHT-XGB can achieve better prediction performance, such as $80.71 \%$ of accuracy rate, 0.614 of MCC. This work shows that the imbalance treatment strategy can improve the prediction of protein-protein interaction sites, and the prediction results is of great significance for understanding life activities and cell activity.

Author Contributions: Conceptualization, B.W. and P.C.; methodology, A.D.; formal analysis, W.W.; data curation, H.Z. and W.W.; writing-original draft preparation, H.Z. and A.D.; writing-review and editing, J.Z. and P.C.; supervision, B.W. and D.F.; funding acquisition, B.W. and D.F. All authors have read and agreed to the published version of the manuscript.

Funding: This research was funded by the National Natural Science Foundation of China (Nos. 61472282, 61672035, and 61872004), Educational Commission of Anhui Province (No. KJ2019ZD05), Open Fund from Key Laboratory of Metallurgical Emission Reduction \& Resources Recycling(KF2017-02), Co-Innovation Center for Information Supply \& Assurance Technology in AHU (ADXXBZ201705), and Anhui Scientific Research Foundation for Returnees.

Conflicts of Interest: All the authors declare no conflict of interest. 


\section{References}

1. Chelliah, V.; Chen, L.; Blundell, T.L.; Lovell, S.C. Distinguishing structural and functional restraints in evolution in order to identify interaction sites. J. Mol. Biol. 2004, 342, 1487-1504. [CrossRef] [PubMed]

2. Nooren, I.M.; Thornton, J.M. Diversity of protein-protein interactions. EMBO J. 2003, 22, 3486-3492. [CrossRef] [PubMed]

3. Hu, S.; Xia, D.; Su, B.; Chen, P.; Wang, B.; Li, J. A Convolutional Neural Network System to Discriminate Drug-Target Interactions. IEEE/ACM Trans. Comput. Biol. Bioinform. 2019. [CrossRef] [PubMed]

4. Patel, T.; Pillay, M.; Jawa, R.; Liao, L. Information of binding sites improves prediction of protein-protein interaction. In Proceedings of the 2006 5th International Conference on Machine Learning and Applications (ICMLA'06), Orlando, FL, USA, 14-16 December 2006; pp. 205-212.

5. Wang, Y.; Mei, C.; Zhou, Y.; Zheng, C.; Zhen, X.; Xiong, Y.; Wang, Y.; Chen, P.; Zhang, J.; Wang, B. Semi-supervised prediction of protein interaction sites from unlabeled sample information. BMC Bioinform. 2019, 20, 699. [CrossRef]

6. Wang, B.; Wang, L.; Zheng, C.-H.; Xiong, Y. Imbalance Data Processing Strategy for Protein Interaction Sites Prediction. IEEE/ACM Trans. Comput. Biol. Bioinform. 2019. [CrossRef]

7. Wei, P.J.; Zhang, D.; Xia, J.; Zheng, C.H. LNDriver: Identifying driver genes by integrating mutation and expression data based on gene-gene interaction network. BMC Bioinform. 2016, 17, 467. [CrossRef]

8. Peng, C.; Liu, C.; Burge, L. DomSVR: Domain boundary prediction with support vector regression from sequence information alone. Amino Acids 2010, 39, 713-726.

9. Sriwastava, B.K.; Basu, S.; Maulik, U. Protein-Protein interaction site prediction in Homo sapiens and E. coli using an interaction-affinity based membership function in fuzzy SVM. J. Biosci. 2015, 40, 809-818. [CrossRef]

10. Daberdaku, S.; Ferrari, C. Exploring the potential of 3D Zernike descriptors and SVM for protein-protein interface prediction. BMC Bioinform. 2018, 19, 35. [CrossRef]

11. Liu, Q.; Chen, P.; Wang, B.; Zhang, J.; Li, J. Hot spot prediction in protein-protein interactions by an ensemble system. BMC Syst. Biol. 2018, 12, 132. [CrossRef]

12. Saethang, T.; Payne, D.M.; Avihingsanon, Y.; Pisitkun, T. A machine learning strategy for predicting localization of post-translational modification sites in protein-protein interacting regions. BMC Bioinform. 2016, 17, 307. [CrossRef] [PubMed]

13. Sriwastava, B.K.; Basu, S.; Maulik, U.; Plewczynski, D. PPIcons: Identification of protein-protein interaction sites in selected organisms. J. Mol. Model. 2013, 19, 4059-4070. [CrossRef] [PubMed]

14. Wang, K.; Gao, J.; Shen, S.; Tuszynski, J.A.; Ruan, J.; Hu, G. An accurate method for prediction of protein-ligand binding site on protein surface using SVM and statistical depth function. BioMed Res. Int. 2013, 2013, 409658. [CrossRef] [PubMed]

15. Zhong, Y.; Guo, Y.; Luo, J.; Pu, X.; Li, M. Effective identification of kinase-specific phosphorylation sites based on domain-domain interactions. Chem. Intell. Lab. Syst. 2014, 136, 97-103. [CrossRef]

16. Fan, W.; Xu, X.; Shen, Y.; Feng, H.; Li, A.; Wang, M. Prediction of protein kinase-specific phosphorylation sites in hierarchical structure using functional information and random forest. Amino Acids 2014, 46, 1069-1078. [CrossRef]

17. Hu, S.S.; Peng, C.; Bing, W.; Li, J. Protein binding hot spots prediction from sequence only by a new ensemble learning method. Amino Acids 2017, 49, 1773-1785. [CrossRef]

18. Guo, H.; Liu, B.; Cai, D.; Lu, T. Predicting protein-protein interaction sites using modified support vector machine. Int. J. Mach. Learn. Cybern. 2018, 9, 393-398. [CrossRef]

19. Wang, B.; Chen, P.; Wang, P.; Zhao, G.; Zhang, X. Radial basis function neural network ensemble for predicting protein-protein interaction sites in heterocomplexes. Protein Pept. Lett. 2010, 17, 1111-1116. [CrossRef]

20. Li, H.; Pi, D.; Wang, C. The prediction of protein-protein interaction sites based on RBF classifier improved by SMOTE. Math. Probl. Eng. 2014, 2014, 528767. [CrossRef]

21. Liu, X.-Y.; Wu, J.; Zhou, Z.-H. Exploratory undersampling for class-imbalance learning. IEEE Trans. Syst. Man Cybern. Part B 2008, 39, 539-550.

22. Wang, B.; Huang, D.-S.; Jiang, C. A new strategy for protein interface identification using manifold learning method. IEEE Trans. Nanobiosci. 2014, 13, 118-123. [CrossRef] [PubMed] 
23. Chen, T.; Guestrin, C. Xgboost: A scalable tree boosting system. In Proceedings of the 22nd ACM SIGKDD International Conference on Knowledge Discovery and Data Mining (KDD 2016), San Francisco, CA, USA, 13-17 August 2016; pp. 785-794.

24. Wang, B.; Chen, P.; Huang, D.-S.; Li, J.-J.; Lok, T.-M.; Lyu, M.R. Predicting protein interaction sites from residue spatial sequence profile and evolution rate. Febs Lett. 2006, 580, 380-384. [CrossRef] [PubMed]

25. Kuo, T.H.; Li, K.B. Predicting Protein-Protein Interaction Sites Using Sequence Descriptors and Site Propensity of Neighboring Amino Acids. Int. J. Mol. Sci. 2016, 17, 1788. [CrossRef] [PubMed]

26. Liu, R.; Jiang, W.; Zhou, Y. Identifying protein-protein interaction sites in transient complexes with temperature factor, sequence profile and accessible surface area. Amino Acids 2010, 38, 263-270. [CrossRef] [PubMed]

27. Mei, C.; Wang, Y.; Lu, K.; Wang, B.; Chen, P. Unbalance Data Processing Strategy for Protein Interaction Sites Prediction. In Proceedings of the 2018 9th International Conference on Information Technology in Medicine and Education (ITME), Hangzhou, China, 19-21 October 2018; pp. 313-317.

28. Dhole, K.; Singh, G.; Pai, P.P.; Mondal, S. Sequence-based prediction of protein-protein interaction sites with L1-logreg classifier. J. Theor. Biol. 2014, 348, 47-54. [CrossRef]

29. Murakami, Y.; Mizuguchi, K. Applying the Naive Bayes classifier with kernel density estimation to the prediction of protein-protein interaction sites. Bioinformatics 2010, 26, 1841-1848. [CrossRef]

30. Singh, G.; Dhole, K.; Pai, P.P.; Mondal, S. Springs: Prediction of protein-protein interaction sites using artificial neural networks. PeerJ PrePrints 2014, 2, e266v2.

31. Porollo, A.; Meller, J. Prediction-based fingerprints of protein-protein interactions. Proteins 2007, 66, 630-645. [CrossRef]

32. Zhang, J.; Kurgan, L. SCRIBER: Accurate and partner type-specific prediction of protein-binding residues from proteins sequences. Bioinformatics 2019, 35, i343-i353. [CrossRef]

33. Ofran, Y.; Rost, B. ISIS: Interaction sites identified from sequence. Bioinformatics 2007, 23, e13-e16. [CrossRef]

34. Hou, Q.; de Geest, P.F.G.; Vranken, W.F.; Heringa, J.; Feenstra, K.A. Seeing the trees through the forest: Sequence-based homo- and heteromeric protein-protein interaction sites prediction using random forest. Bioinformatics 2017, 33, 1479-1487. [CrossRef] [PubMed]

35. Zeng, M.; Zhang, F.; Wu, F.X.; Li, Y.; Wang, J.; Li, M. Protein-protein interaction site prediction through combining local and global features with deep neural networks. Bioinformatics 2020, 36, 1114-1120. [CrossRef] [PubMed]

36. Wei, Z.-S.; Han, K.; Yang, J.-Y.; Shen, H.-B.; Yu, D.-J. Protein-protein interaction sites prediction by ensembling svm and sample-weighted random forests. Neurocomputing 2016, 193, 201-212. [CrossRef]

37. Li, Y.; Ilie, L. DELPHI: Accurate deep ensemble model for protein interaction sites prediction. bioRxiv 2020. [CrossRef]

38. Bonvin, A.M. Flexible protein-protein docking. Curr. Opin. Struct. Biol. 2006, 16, 194-200. [CrossRef]

39. Ansari, S.; Helms, V. Statistical analysis of predominantly transient protein-protein interfaces. Proteins 2005, 61,344-355. [CrossRef]

40. Fariselli, P.; Pazos, F.; Valencia, A.; Casadio, R. Prediction of protein-protein interaction sites in heterocomplexes with neural networks. Eur. J. Biochem. 2002, 269, 1356-1361. [CrossRef]

41. Glaser, F.; Pupko, T.; Paz, I.; Bell, R.E.; Bechor-Shental, D.; Martz, E.; Ben-Tal, N. ConSurf: Identification of functional regions in proteins by surface-mapping of phylogenetic information. Bioinformatics 2003, 19, 163-164. [CrossRef]

42. Smith, M.R.; Martinez, T.; Giraud-Carrier, C. An instance level analysis of data complexity. Mach. Learn. 2014, 95, 225-256. [CrossRef]

43. Breiman, L. Random forests. Mach. Learn. 2001, 45, 5-32. [CrossRef]

44. Bahety, A. Extension and evaluation of id3-decision tree algorithm. Entropy 2014, 2, 1-8.

45. Verdikha, N.A.; Adji, T.B.; Permanasari, A.E. Study of Undersampling Method: Instance Hardness Threshold with Various Estimators for Hate Speech Classification. IJITEE 2018, 2, 39-44. [CrossRef]

46. Wilson, D.L. Asymptotic properties of nearest neighbor rules using edited data. IEEE Trans. Syst. Man Cybern. 1972, SMC-2, 408-421. [CrossRef]

(C) 2020 by the authors. Licensee MDPI, Basel, Switzerland. This article is an open access article distributed under the terms and conditions of the Creative Commons Attribution (CC BY) license (http://creativecommons.org/licenses/by/4.0/). 